\title{
Pacific Northwest Center for Cryo-EM: Coast-to-coast Training and CryoTEM Services
}

Claudia Lopez ${ }^{1}$, Craig Yoshioka ${ }^{1}$ and Lauren Hales Beck ${ }^{2}$

${ }^{1}$ Oregon Health \& Science University, Portland, Oregon, United States, ${ }^{2}$ Pacific Northwest cryoEM Center, Portland, Oregon, United States

The Pacific Northwest Center for Cryo-EM (PNCC) [1] is a state-of-the-art electron microscopy facility funded by the NIH Common Fund [2] and operated jointly by Oregon Health \& Science University (OHSU) [3] and the Pacific Northwest National Laboratory (PNNL) [4]. The PNCC mission is to service researchers nationwide by helping them tackle challenging scientific problems using cryo electron microscopy. PNCC offers users a seamless workflow from sample analysis and optimization to grid preparation, automated high-resolution micrograph collection, image analysis, 3D reconstruction and model construction and validation [1]. Multiple levels of service and access are available and correspond with the type of analysis desired. Individuals wanting access to PNCC resources need to submit their research proposals through our User Portal [5]. We have two different type of proposal available to users: limited access for one day of microscope access and general access with up to 20 days per year for two years. Both Single Particle Analysis (SPA) and tomography projects are welcome. Group workshops are held annually, they introduce new users to cryo-EM methodology and theory and are open to the scientific community [6]. Our group training workshops consist of sample preparation, microscope operation, automated data acquisition (EPU and SerialEM) and image processing. The microscope operation workshop offered at PNCC is designed to provide independence to those researchers wanting to operate the microscopes at their home institutions or the ones available at PNCC. Onsite individual training is provided only for users with approved projects at PNCC and is tailored to the specific samples, instruments, and methods of interest. Internships at PNCC are also a great opportunity for lab managers or senior staff to consolidate their training and provide a solid foundation for professional development [5]. PNCC instrument time, consumables, and staff effort are provided to users at no-cost after independent peer-review of proposals. Among PNCC's capabilities [7] are: One Talos Arctica with standalone K2, two Titan Krios with standalone K3 and Falcon 3, and two Titan Krios with Falcon 3 and post GIF K3. Our low humidity wet lab is currently equipped with a Vitrobot Mk IV and Leica GP2 vitrification units. A Chameleon sample preparation device will be installed in 2021 [8].

References

[1] https://pncc.labworks.org/

[2] https://commonfund.nih.gov/CryoEM

[3] https://www.ohsu.edu/xd/

[4] https://www.pnnl.gov/

[5] https://eus.emsl.pnl.gov/Portal/

[6] https://pncc.labworks.org/training

[7] https://pncc.labworks.org/instruments

[8] The author acknowledges the support of the National NIH-Funded Cryo-EM Centers: Transformative High Resolution Cryo-Electron Microscopy (grant number U24GM129547) 